Abstract 3 Table 2 Relative interaction of atrial update, transthyretin cardiac amyloidosis (ATTR-CA) and atrial fibrillation (AF). Caption: Total incudes total number of patients between the 2 groups

\begin{tabular}{|c|c|c|c|c|c|c|c|c|}
\hline \multirow{2}{*}{$\begin{array}{c}\text { Total Cohort } \\
\text { N=580 }\end{array}$} & \multicolumn{5}{|c|}{ Atrial Uptake(+) } & \multicolumn{4}{c|}{ Atrial Uptake(-) } \\
\hline ATTR-CA(-) & 58 & $10 \%$ & 51 & $9 \%$ & 358 & $62 \%$ & 146 & $25 \%$ \\
\hline ATTR-CA(+) & 59 & $10 \%$ & 56 & $10 \%$ & 105 & $18 \%$ & 43 & $7 \%$ \\
\hline
\end{tabular}

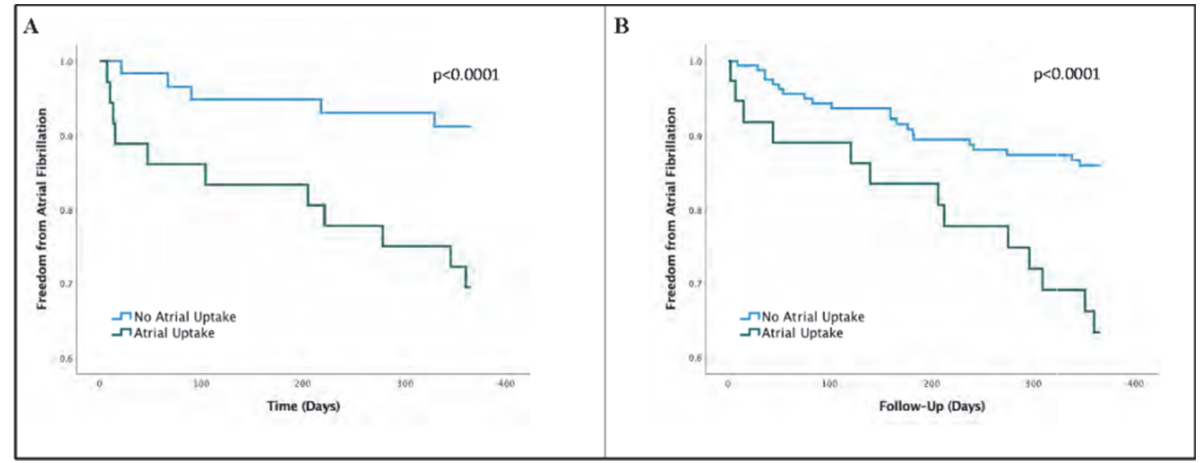

Abstract 3 Figure 2 Freedom from atrial fibrillation in patients with and without atrial uptake

CA and AF. Of 117 patients with AU, 107 (91\%) had AF. In contrast, of 463 patients without AU 189 (41\%) had AF. AU was associated with a significantly higher incidence of $\mathrm{AF}$ in those with and without ATTR-CA $(\mathrm{p}<0.001)$. On Cox proportional hazards analyses, freedom from $\mathrm{AF}$ at 1-year in patients with ATTR-CA was significantly lower in patients with AU (HR: 2.27; 95\% CI: 1.37-3.78; p<0.0001) (figure 2). Similarly, freedom from $\mathrm{AF}$ at 1 -year in patients without ATTR-CA was significantly lower in patients with AU (HR: 2.21; 95\% CI: 1.46-3.34; p<0.0001). On Kaplan-Meier survival analysis, there were no significant differences in mortality rates in patients with AF and ATTR-CA; with AU vs. without AU (all-cause mortality, $\mathrm{p}=0.79$; 1-year mortality, $\mathrm{p}=0.76$ ). There were no significant differences in mortality rates in patients with AF and without ATTR-CA; with AU vs. without AU (all-cause mortality, $p=0.20 ; 1$-year mortality, $p=0.57$ ). For the first time, we described that the presence of $\mathrm{AU}$ was independent of ATTR-CA diagnosis and was associated with higher rates of AF. AU was not associated with increased mortality in those with AF. This leads to the question of the role of $\mathrm{AU}$ in $\mathrm{AF}$ diagnosis/management and the interaction of $\mathrm{AU}$ with ATTR-CA diagnosis.

\section{PREDICTIVE GENETIC TESTING IN INHERITED CARDIAC CONDITIONS: FINDINGS FROM A LARGE IRISH COHORT}

1J Murphy, ${ }^{1} \mathrm{C}$ Kirk, ${ }^{2} \mathrm{G}$ Abboud Guerra, ${ }^{3} \mathrm{~J}$ Galvin, ${ }^{4} \mathrm{D}$ Ward, ${ }^{5} \mathrm{~T}$ Prendiville, ${ }^{3} \mathrm{C}$ McGorrian, ${ }^{5}$ SA Lynch. 'University College Dublin, Ireland; ${ }^{2}$ St Vincent's University Hospital, Ireland; ${ }^{3}$ Mater University Hospital, Ireland; ${ }^{4}$ Tallaght University Hospital, Dublin, Ireland; ${ }^{5}$ Children's Health Ireland Crumlin, Dublin, Ireland

10.1136/heartjnl-2021-ICS.4
Introduction Inherited cardiac conditions comprising cardiomyopathies (hypertrophic, dilated, arrhythmogenic) and cardiac ion channelopathies (long QT/Brugada syndromes, CPVT) predispose to sudden cardiac death. Predictive genetic testing of at-risk family members informs patient management and is cost-effective as those who test negative can be discharged from lifelong cardiac follow-up. Aim: Through predictive testing uptake, investigate the demographics and genetic contribution of inherited cardiac conditions in the Republic of Ireland. Methods Predictive testing data from 2003 to 2020 was collated through interrogation of departmental databases and molecular genetic reports at a national genetics centre and two tertiary cardiac referral clinics.

Results Collectively, 1,535 predictive tests for pathogenic/likely pathogenic variants were undertaken in 1,508 individuals from 241 families, including 27 individuals tested for two familial variants. The median age at testing was 36 years (range $0.07-$ 90 years). From 1,152 adults, more females (58\%) presented for testing than males (42\%). Amongst 365 children, marginally more boys (53\%) were tested than girls (47\%). On average, six individuals per family were tested (range 1 - 84). Seven hundred and nineteen individuals (48\%) were found to be genotype-positive for their familial variant(s). Overall, predictive testing was performed for 26 genes. Testing for long QT syndrome (LQTS) and hypertrophic cardiomyopathy (HCM) genes represented $48 \%$ and $29 \%$ of the cohort, respectively. Other cardiomyopathies and arrhythmia syndromes accounted for $10 \%$ and $8 \%$ respectively, with $5 \%$ tested for syndromic disease genes. The most frequently tested gene was KCNQ1 (LQTS; 24\% of tests), followed by MYBPC3 (HCM/DCM; 20\%), KCNH2 (LQTS; 17\%), MYH7 (HCM/DCM; 8\%) and SCN5A (LQT/Brugada syndromes; $8 \%)$. There was an absence of testing for several sarcomeric 
genes (MYL3, TPM1, ACTC1), in addition to the desmosomal genes DSG2, DSC2 and JUP.

Conclusion Predictive testing has potentially allowed up to 789 genotype-negative individuals (and their offspring) to be reassured and discharged from long term cardiac follow-up. Our data suggests adult females are more forthcoming for predictive testing than their male counterparts. The absence of testing for several cardiomyopathy genes suggests low frequency or low penetrance of these variants in the Irish population. The large size of families in our cohort represents an opportunity to develop gene penetrance and genotype-phenotype correlation data to assist in clinical management of genotype-positive individuals.

\section{Oral abstracts}

\section{COMPARISON OF NEXT GENERATION P2Y12 INHIBITORS TO CLOPIDOGREL IN PATIENTS WITH ACUTE CORONARY SYNDROME: AN ANALYSIS FROM THE BRITISH CARDIOVASCULAR INTERVENTION SOCIETY DATABASE OF 382,361 'REAL-WORLD' PATIENTS}

S Saluja, H Contractor, F Mannan, J Sarma, S Saluja, S Garg, S Anderson. University Hospital of South Manchester, Manchester, UK

\subsection{6/heartjnl-2021-ICS.5}

Objective and Background Dual antiplatelet therapy is standard of care in patients with acute coronary syndrome (ACS) treated with percutaneous coronary intervention (PCI), however debate remains regarding the optimal P2Y12 receptor antagonist to combine with aspirin.

Methods We performed a retrospective analysis of all PCI procedures for ACS recorded in the British Cardiovascular Intervention Society database between 2007 and 2014 treated with aspirin and either clopidogrel, prasugrel or ticagrelor. The primary outcome measure was 30-day all-cause mortality, with secondary outcomes of mortality at 1 and 5 years. Odds ratios (OR) for mortality were determined from multivariable logistic regression. We used multiple imputations to address missing values and reduce inherent bias from complete case-only analysis. Difference in outcome amongst patients receiving different P2Y12 inhibitors was evaluated by Kaplan-Meier survival curves and log-rank testing. All statistical analysis, including calculation of marginal effects, was performed using SPSS version 21 and $\mathrm{R}$ software version 2.13.1.

Results Among 382,361 eligible patients with 2 million person-years of observation, $6.8 \%(n=26,000)$ of patients had ticagrelor, $8.0 \% \quad(\mathrm{n}=30,590)$ had prasugrel and $85.2 \%$ $(n=325,771)$ were treated with clopidogrel for ACS. Mean age of participants was $62.8 \pm 4.3$ years; $77.1 \%$ of patients were males and $31.6 \%$ had diabetes. The proportion of people undergoing intervention for stable angina was largest and comprised of 164,797 participants, followed by NSTEMI [157,532 participants] with only a small proportion of patients undergoing intervention for a STEMI [60,032 participants]. ACS patients treated with clopidogrel were slightly older and had a higher percentage of people who had renal failure, but a lower proportion in this group were treated for a STEMI. This reflects on the increasing provision for PCI for STEMI during years when prasugrel and ticagrelor were introduced as options. Crude mortality rates for ACS

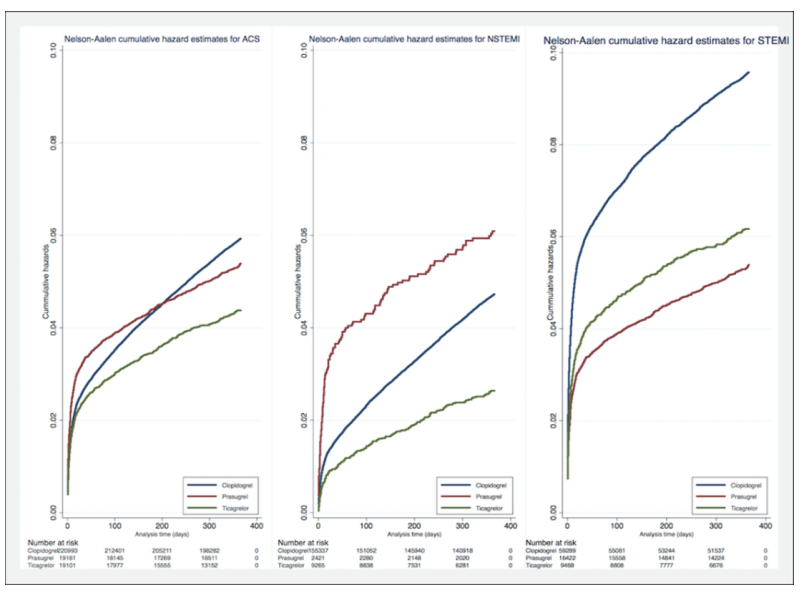

Abstract 5 Figure 1 Cumulative incidence of all-cause mortality over 1 year showing (A) adjusted rates in all ACS patients treated with either clopidogrel, ticagrelor or prasugrel (B) adjusted rates in all NSTEMI patients treated with either clopidogrel, prasugrel or ticagrelor (adjusted rates in STEMI patients treated with clopidogrel, prasugrel, or ticagrelor. HR: hazard ratio; $\mathrm{Cl}$ : confidence intervals

were $34.7,31.6$ and 30.9 deaths per 1000-person-years for patients receiving clopidogrel, prasugrel, and ticagrelor, respectively. In age-sex unadjusted multinomial logistic regression analysis, use of ticagrelor or prasugrel rather than clopidogrel led to respective reductions in 1-year mortality of 64\% [OR 0.34, 95\% CI (0.32-0.36)], and 27\% (OR 0.73 $(0.69-0.77), p<0.0001)$. Using clopidogrel as the reference, the age-sex adjusted 1-year mortality rate was 63\% [(OR

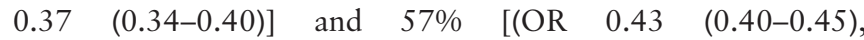
$\mathrm{p}<0.0001)$ ] lower with ticagrelor and prasugrel, respectively in ST-elevation myocardial infarction (STEMI) patients and $80 \% \quad[(\mathrm{OR} \quad 0.20(0.18-0.23), \mathrm{p}<0.0001)]$ and $36 \% \quad[(\mathrm{OR}$ $0.43(0.40-0.45), \mathrm{p}<0.0001)]$ lower in non-STEMI patients. Furthermore, using marginal effects, we demonstrate that while the probability of mortality increases with increasing age and BMI, it is lower across all ages and BMIs for patients on ticagrelor compared to patients on prasugrel. Figure 1 demonstrates the cumulative incidence for all-cause mortality stratified by antiplatelets.

Conclusions This very large, real-world dataset of patients presenting with ACS demonstrates a significant net clinical benefit favouring the use of ticagrelor and prasugrel over clopidogrel in ACS patients for DAPT. This analysis concurs with the data from the landmark TRITON and PLATO RCTs, suggesting these agents should be considered as the standard of care in the management of ACS.

\section{DEMOGRAPHIC, CLINICAL AND ANGIOGRAPHIC PROFILE OF YOUNG ADULTS ( $\leq 45$ YEARS) UNDERGOING PERCUTANEOUS CORONARY INTERVENTION FOR ST ELEVATION MYOCARDIAL INFARCTION}

${ }^{1} \mathrm{C}$ McCaughey, ${ }^{1} \mathrm{R}$ Kumar, 'S Teehan, ${ }^{1} \mathrm{R}$ Gardiner, ${ }^{1} \mathrm{~J}$ Kumar, ${ }^{1} \mathrm{C}$ Murray, ${ }^{2} \mathrm{~B}$ Khan, 'G Murphy. ${ }^{1} \mathrm{C}$ Daly; ${ }^{1}$ St James's Hospital Dublin, Ireland; 'Trinity College Dublin, Ireland

\subsection{6/heartjnl-2021-ICS.6}

Introduction Premature (defined as $\leq 45$ years old at presentation) ST Elevation Myocardial Infarction (STEMI) remains a significant population health issue despite overall reductions in 